\title{
Modeling of Pocket Implanted MOSFETs for Anomalous Analog Behavior
}

\author{
Kanyu Mark Cao, Weidong Liu, Xiaodong Jin, Karthik Vasanth", Keith Green ${ }^{*}$, John Krick*, \\ Tom Vrotsos", and Chenming $\mathrm{Hu}$ \\ Department of Electrical Engineering and Computer Sciences, University of California, Berkeley, CA 94720, USA \\ Tel: 510-643-2639 Fax: 510-643-2636 Email: kcao@cory.eecs.berkeley.edu \\ "Spice Modeling Laboratory, Texas Instruments, Dallas, TX 75266, USA
}

\begin{abstract}
Pocket implant is widely used in deep-sub-micron CMOS technologies to combat short channel effects. It, however, brings anomalously large drain-induced threshold voltage shift and low output resistance to long channel devices. This creates a serious problem for high-performance analog circuits. In this paper, the first physical model of these effects are proposed and verified against data from a $0.18 \mu \mathrm{m}$ technology. This model is suitable for SPICE modeling.
\end{abstract}

\section{Introduction}

Pocket implant is widely used in deep sub-micron CMOS technologies to reduce $V_{T}$ roll-off and punch-through [1]. This technique, however, produces large drain-induced $V_{T}$ shift and low $R_{\text {out }}$ in long channel devices [2], greatly affecting analog circuit design and performance. Physical compact model for these effects, however, is not available.

In this work, we report the first physical model of these effects suitable for compact MOSFET modeling. The proposed model is verified against both simulation and experimental data from a $0.18 \mu \mathrm{m}$ CMOS technology.

Drain-induced threshold voltage shift (DITS)

Fig. 1 illustrates the enhanced DITS effect in long channel devices with pocket implants compared with uniformly doped devices using 2-D simulation. Fig. 2 is an illustration of the structure used in the device simulation. DITS increases by 2 to 3 times for long channel devices. Fig. 3 shows the simulated $\varphi_{s}$ vs. $x$ for two $V_{d s}$ biases for a pocket implanted device. Clearly, $\varphi_{s}$ is independent of $x$ in the center segment of the channel and the drain barrier peak decreases with increasing $V_{d s}$ thus leading to DITS. Starting with the drift-diffusion equation, we derived a drain current expression by considering the source-end, center, and drain-end sections of the channel separately and applying a DIBL model [3] to the drainside barrier only:

$$
I_{d s}=\frac{q W D_{n} N_{D}\left(1-e^{-\beta V_{d s}}\right)}{\int_{0}^{L} e^{-\beta\left(\varphi_{s}-\varphi_{s \text { satre }}\right)} d y} \cdot \frac{\varepsilon_{s i} T_{o x}}{\beta \varepsilon_{o x}\left(V_{T 0}-V_{F B}-2 \varphi_{B}\right)}=A W \frac{1-e^{-\beta V_{d t}}}{C_{i} L+\left(1+e^{-C_{1} V_{d t}}\right)}(1)
$$

where $\beta=q / k T, \varphi_{s}$ is the surface potential, and $C_{1}$ and $C_{2}$ as

$$
\begin{aligned}
& C_{1}=e^{-\beta\left(\varphi_{s 2}-\varphi_{\min }\right)} \sqrt{\beta\left[q N_{p} X_{d e p}-\varepsilon_{o x}\left(V_{T 0}-V_{F B}-2 \varphi_{B}\right) / T_{o x}\right] / \varepsilon_{s i} X_{d e p} \pi}, \\
& C_{2}=\beta \cdot\left(e^{-L_{p} / 2 l_{p}}+2 e^{-L_{p} / l_{p}}\right)
\end{aligned}
$$

are model parameters. $l_{p}=\sqrt{\varepsilon_{s i} T_{o x} X_{d e p} / \varepsilon_{o x}}$. If $V_{T}$ is defined to be the gate voltage at which $I_{d s}=I_{c r i t}=I_{T} \cdot W / L$, where $I_{T}$ is the threshold current chosen experimentally. Then the threshold voltage shift can be derived as:

$$
\Delta V_{T}=-S \cdot \log \left(\frac{I_{d s}}{I_{c r i t}}\right)=-S \cdot \log \left(\frac{\left(1-e^{-\beta V_{d s}}\right)}{1+\left(1+e^{-C_{2} V_{d t}}\right) / C_{1} L}\right)
$$

where $S$ is the sub-threshold swing and can be calculated or extracted [4].

\section{Modeling the output resistance}

Pocket implants also lower the output resistance, $R_{\text {our }}$. Figure 4 shows that at low $V_{g s}, R_{\text {out }}$ can be 10 to 100 times smaller because of the pocket implant. Pocket implants affect output resistance in two ways. Firstly, DITS causes $I_{d s}$ to increase with increasing $V_{d s}$. The early voltage due to this mechanism can be derived from (1):

$$
V_{A, D I T S}=I_{d s} \cdot\left(\frac{d I_{d s}}{d V_{d s}}\right)^{-1}=\frac{\left(C_{1} L+1\right) e^{C_{2} V_{d t}+1}}{C_{2}}
$$

Since parameters $C_{I}$ and $C_{2}$ in (1) are used to model $I_{d s}$ in subthreshold-threshold region, we introduce parameters $C_{0 c}$, $C_{l c}$, and $C_{2 c}$ to accurately capture this effect in strong inversion region:

$$
V_{A, D r r s}=C_{0 c} \cdot \frac{\left(C_{1 c} L+1\right) e^{C_{2 c} v_{d s}+1}}{C_{2 c}}
$$

The second effect of the pocket implant on $R_{\text {out }}$ is that the output resistance due to all mechanisms (CLM, DIBL, etc.) is reduced by a factor that varies with $V_{g s}$ and $L$. Let us compare a pocket-implanted device with a MOSFET uniformly doped to the pocket concentration, $N_{p}$. In Figure 5, both devices are partitioned into two parts. The device on top has a length $L_{p}$, the length of the pocket. The lower part is the rest of the channel with length $L-L_{p}$. This partition is useful because in the saturation region, $R_{\text {out }}$ is mainly determined by the effects in a small region close to the drain, and the rest of the channel, i.e. the lower device may be considered simply a source resistance. For the cascode circuit in Fig. 5(a), the output resistance in saturation is well known:

$$
R_{\text {out }}=\left(g_{m 1} r_{o 2}+1\right) \cdot r_{o 1}=\left(L / L_{p}\right) \cdot r_{o 1}
$$

where $r_{o 1}$ is the output resistance of the device on top. In a similar way, $\mathbf{R}_{\text {out }}$ of the pocket device, i.e. the circuit in Fig. 5(b) can be derived as:

$$
R_{\text {outp }}=\left(g_{m i} r_{\text {or } 2 p}+1\right) \cdot r_{\text {olp }}=\frac{L}{L_{p}}\left(1-\frac{L-L_{p}}{L}\left(1+\sqrt{\frac{L_{p}}{L}} \sqrt{\frac{\left(V_{g}-V_{T}\right)^{2}}{\delta V_{T}^{2}}-2 \frac{V_{g}-V_{T}}{\delta V_{T}}+\frac{L_{p}}{L}}\right)^{-1}\right) \cdot r_{\text {olp }}
$$

where $\delta V_{T}=V_{T}\left(N_{p}\right)-V_{T}\left(N_{s u b}\right)$. Since $\mathrm{M}_{1}$ and $\mathrm{M}_{1 \mathrm{p}}$ have the same doping and since Early voltage $V_{A}$ is insensitive to $V_{g s}, \mathbf{M}_{1}$ and $\mathrm{M}_{1 \mathrm{p}}$ should have the same $V_{A}$.

$$
\frac{r_{o 1}}{r_{o l p}} \equiv \frac{V_{A} / I_{d s 1}}{V_{A} / I_{d s 1 p}}=\frac{I_{d s 1 p}}{I_{d s 1}}=\frac{\left(V_{g}-V_{T}-V_{p}^{*}\right)^{2}}{\left(V_{g}-V_{T}-V^{*}\right)^{2}}=\left(1+\left(1-\sqrt{L_{p} / L}\right) \cdot \frac{\delta V_{T}}{V_{g}-V_{T}}\right)^{2}(7)
$$


Considering $\delta V_{T} \ll\left(V_{g}-V_{T}\right)$, using equations (5), (6) and (7) the output resistance of the pocket-implanted device can be rewritten as:

$$
R_{\text {outp }}=F \cdot R_{\text {out }}
$$

where $R_{o u t}$ is the output resistance of the uniformly doped device, which can be modeled by conventional models such as BSIM $3 v 3$ [4]. $\mathrm{F}$ is a "degradation factor"

$$
F=\left(1+\sqrt{\frac{L}{L_{P}}} \frac{\delta V_{T}}{\left(V_{s}-V_{T}\right)}\right)^{-1} \cdot\left(1+\left(1-\sqrt{L_{P} / L}\right) \cdot \frac{\delta V_{T}}{V_{g}-V_{T}}\right)^{-2}=\left(1+P_{F} \cdot \sqrt{L} /\left(V_{8}-V_{T}\right)\right)(9)
$$

where $P_{F} \equiv \delta V_{T} / \sqrt{L_{p}}$ should be considered a fitting pa-

rameter decided by the characteristics of pocket implant.

\section{Results and Discussion}

Eq. (3) can be added to a conventional $V_{T}$ model as a new term. Fig.6 shows the agreement between the model and the measured $V_{T}$ of a $W / L=5 \mu \mathrm{m} / 10 \mu \mathrm{m}$ device. Fig. 7 shows that the BSIM3 $V_{T}$ model [3] does not model the $V_{d s}$ dependence of $V_{T}$ at long gate lengths. Fig. 8 shows that the new model significantly improves the fitting using parameters extracted from Fig. 6. The new threshold shift model can give rise to DITS at long channel lengths where the conventional DIBL theory predicts none. This can be explained by the fact that at $V_{g} \sim V_{T}$ and small $I_{d s}$, there is little voltage drop in most of the channel region. Therefore, even in a long channel device, nearly all the $V_{d s}$ is available to reduce the drain-side barrier height as shown in Fig. 3 and the drain barrier height can significantly affect $I_{d s}$.

To verify the $R_{\text {out }}$ model of (9), 2-D simulations and measurements are performed for identical devices with and without pocket implant. Fig. 9 shows that the "degradation factor" is indeed a constant, independent of $\mathrm{V}_{\mathrm{ds}}$ in the saturation region which is in agreement with (9). Our derivation is general and does not depend on what mechanisms determine the $R_{\text {out }}$. From the model, at high $V_{g . s}$ and long $L$, if $F$ is plotted versus $\left(V_{g s}-V_{T}\right) / \sqrt{L}$, all data should fall on a universal curve. This is verified in Fig. $10 . F$ vs. $V_{g s}$ for different $L$ also agrees with the 2-D device simulation data in Fig. 11. In Fig. 12, this

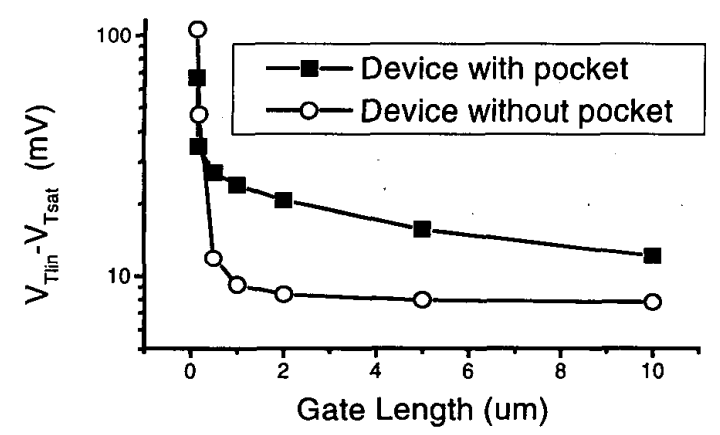

Fig. 1. Comparison of $\left(V_{\text {Tin }}-V_{T s a t}\right)$ vs. $L$ between two devices with and without pocket implant by 2-D simulation. The threshold voltage shift is 2 3 times larger for the pocket implanted device at long gate lengths. model of the degradation factor is compared with the ratio of output resistance measured on devices with and without pocket implants. The devices are otherwise identically fabricated using a $0.18 \mu \mathrm{m}$ process. The agreement is excellent.

The proposed model not only provides the first physical analysis for the anomalous analog behaviors but is also suitable for use in compact modeling of analog devices. It was implemented into the BSIM3 model and model parameters were extracted for a $0.18 \mu \mathrm{m}$ technology. Fig. 13 shows that the output resistance fitting is excellent and the fitting in long channel, low $V_{g s}-V_{T}$ region has been significantly improved.

Both the threshold voltage model and the output resistance model are reduced to the original model when $1 / C_{l}, C_{2 c}$, and $\delta V_{T}$ approach zero. In that case, $\Delta V_{T}=0$ in (3), $V_{A, D r r s}=\infty$ in (4a), and $F=1$ in (9) respectively. Also from the expression, we can see that to minimize the degradation of the output resistance, pocket implant with lower peak concentration and wider lateral length is desired. These are also the conditions to minimize the long channel DITS effect. This agrees with the fact that output resistance degradation and long channel DITS are highly correlated [2]. Although the models are developed for pocket implanted devices, it can also improve the model accuracy of devices without pocket implant if it has reverse short channel effect (RSCE) due to defect-enhanced diffusion.

\section{Acknowledgements}

The authors want to thank Joe Watts and Peiqi Xuan for discussions and BTA for providing model development tool. The work is supported by SRC under contract $98-S J-417$, Compact Modeling Council, and Texas Instruments under the MICRO program.

\section{References}

[1] M.Rodder et al., "A $0.10 \mu \mathrm{m}$ Gate Length CMOS Technology with 30A Gate Dielectric for 1.0V-1.5V Applications," IEDM Tech. Digest, $p$ 223, 1997.

[2] A. Chatterjee et al., "Transistor Design Issues in Integrating Analog Functions with High Performance Digital CMOS," Proc. VLSI Sypm., pp.147, 1999.

[3] Zhi-Hong Liu et al., "Threshold Voltage Model for Deep-Submicrometer MOSFET's," IEEE TED Vol. 40, No. 1, pp. 86, Jan. 1993.

[4] Weidong Liu et al., BSIM3v3.2 MOSFET Model User's Manual (1998). pp. 3-10. http://www-device.eecs.berkeley.edu/ bsim3/.

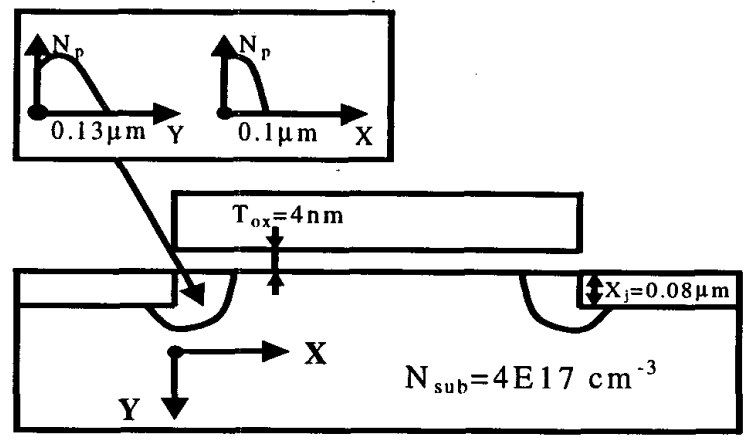

Fig. 2. Device structure used in 2-D device simulations. Pocket doping profile shown simulates that of a $0.18 \mu \mathrm{m}$ technology. 


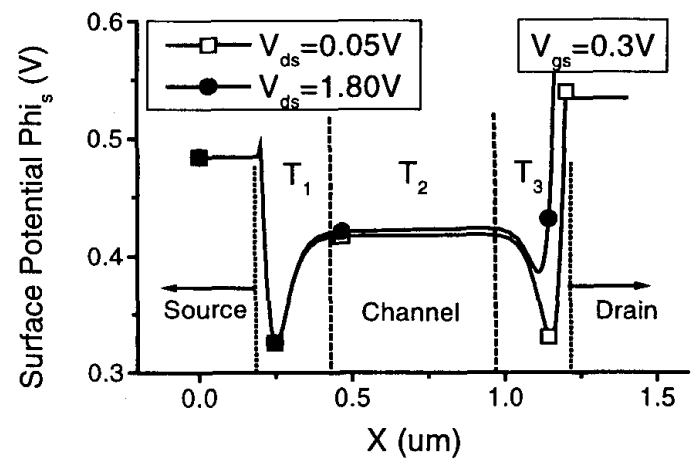

Fig. 3. Simulated channel surface potential. Only the drain barrier is significantly affected by $\mathrm{V}_{\mathrm{ds}}$. The integration in Eq. 1 is carried out in regions $T_{1}, T_{2}$, and $T_{3}$.
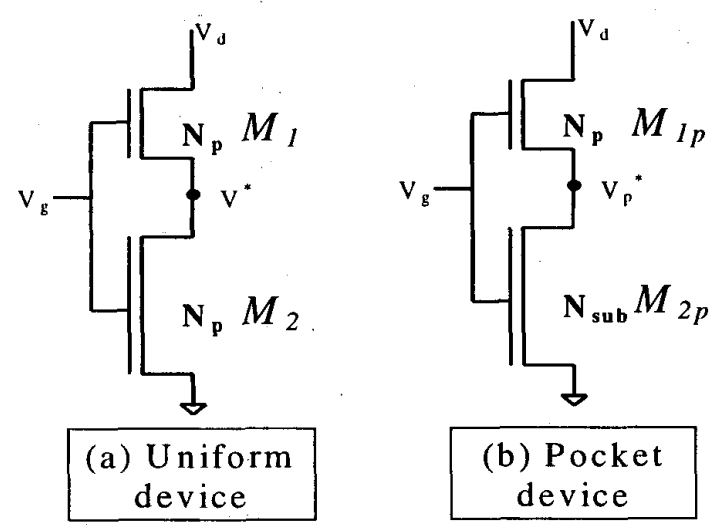

Fig. 5. Equivalent circuits used in the derivation of the output resistance model. A uniformly doped device is shown in (a) and a pocket implanted device in (b).

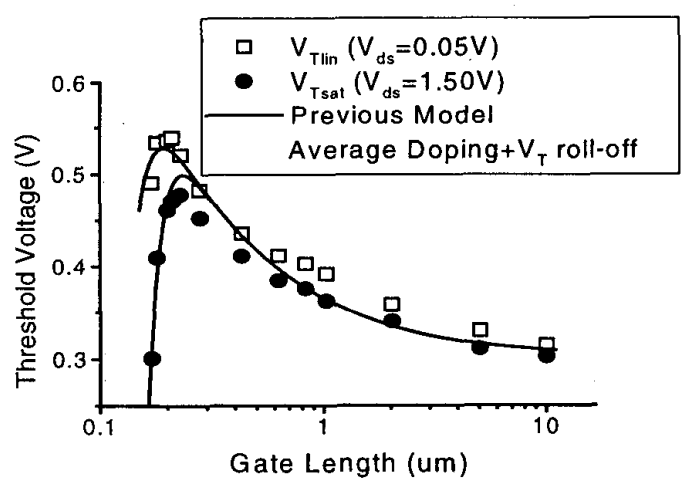

Fig. 7. Best-effort fitting of experimental data with the BSIM3 model. The model shows negligible DIBL effect $\left(V_{d s}\right.$ dependence) at long gate lengths.

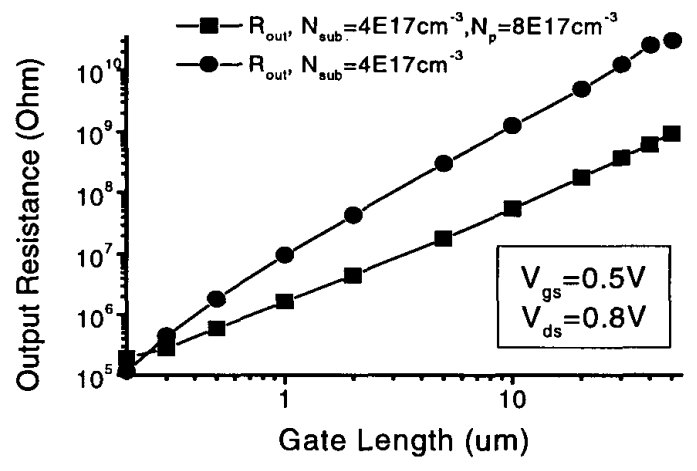

Fig. 4. Simulated $R_{\text {out }}$ of the device with pocket implant is more than 10 times smaller at long channel length. One device has a pocket doping of $\mathrm{N}_{\mathrm{p}}=8 \mathrm{E} 17 \mathrm{~cm}^{-3}$, and pocket length $\mathrm{L}_{\mathrm{p}}=0.08 \mu \mathrm{m}$. The devices are otherwise identical.

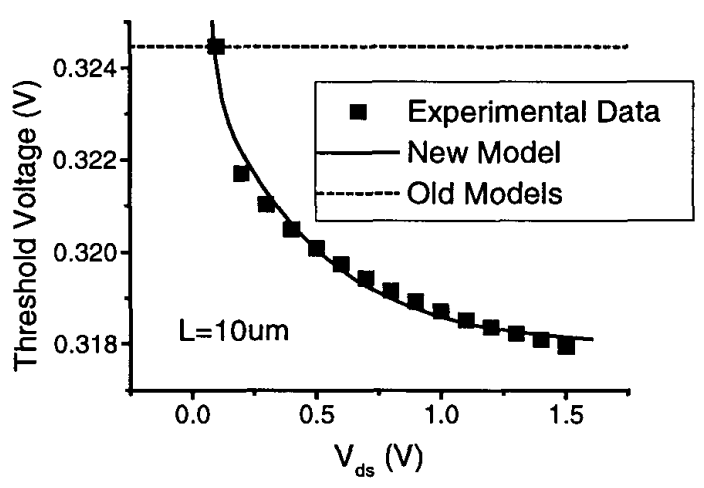

Fig. 6. The proposed model is in excellent agreement with measured $V_{T}$ vs. $V_{d s}$ of a $10 \mu \mathrm{m}$ MOSFET fabricated using a $0.18 \mu \mathrm{m}$ process with pocket implantation.

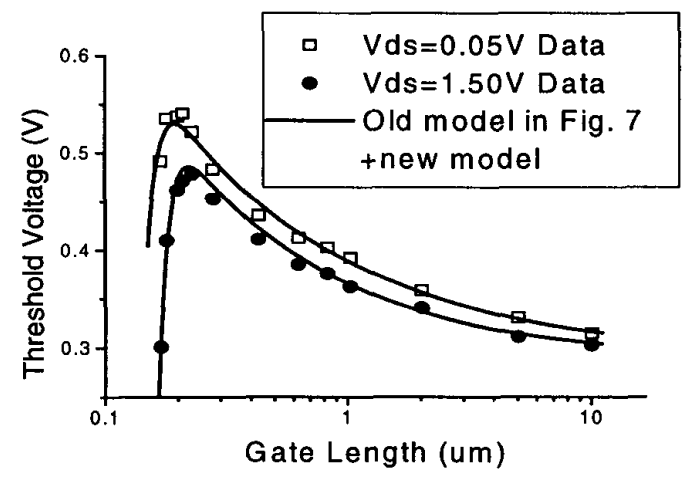

Fig. 8. Fitting of experimental data with the new model added to the BSIM model used in Fig. 7. The number of fitting parameters is the same as in Fig. 7 because parameters extracted in Fig. 6 are used in the new model. 


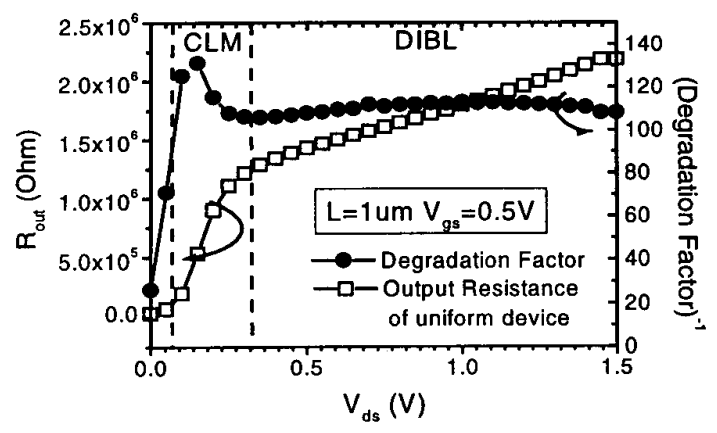

Fig. 9. Degradation Factor of output resistance from 2-D simulation is almost independent of $\mathrm{V}_{\mathrm{ds}}$ as predicted by the proposed model in both channel length modulation (CLM) and drain-induced barrier lowering (DIBL) regions.

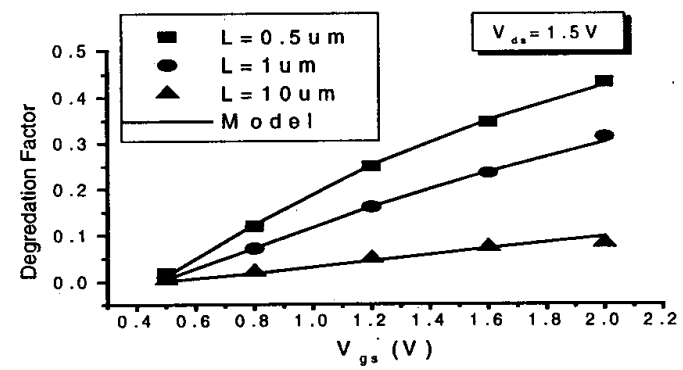

Fig. 11. Agreement of $V_{g s}$ dependence of the degradation factor $F$ between model and simulation for different gate lengths.

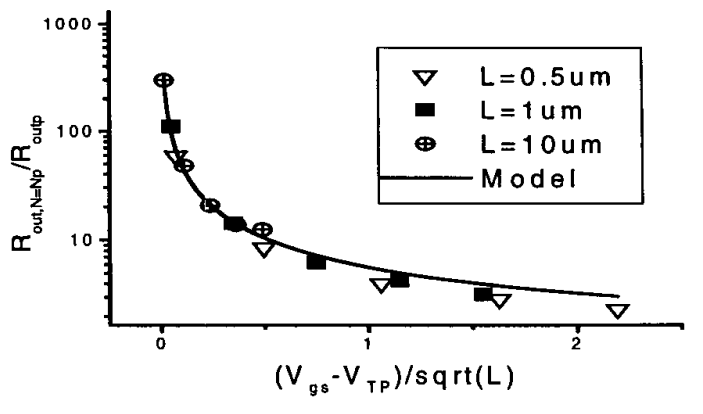

Fig. 10. Simulation verification of the predicted universal curve of the degradation factor (1/F plotted) vs. $\left(V_{g s}-V_{T P}\right) / \sqrt{L}$.

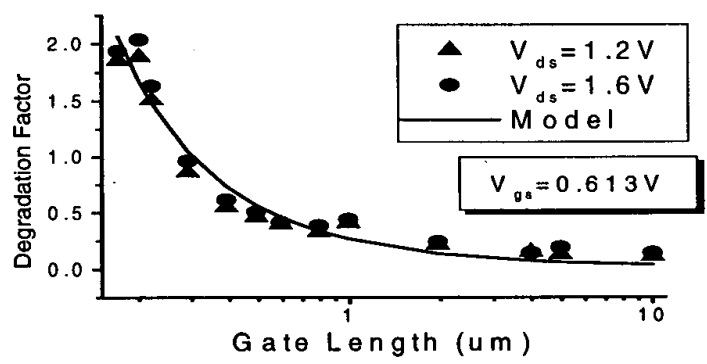

Fig. 12. Agreement of model and experimental data of a $0.18 \mu \mathrm{m}$ process with and without pocket implant. Note that the degradation factor is very different from Fig. $9,10,11$ because the uniform device is doped to the substrate level.
W5L015 1.IV W/L $=5.00 / 0.15 \mathrm{Te27C}$ VB $=0.00 \mathrm{~V}$

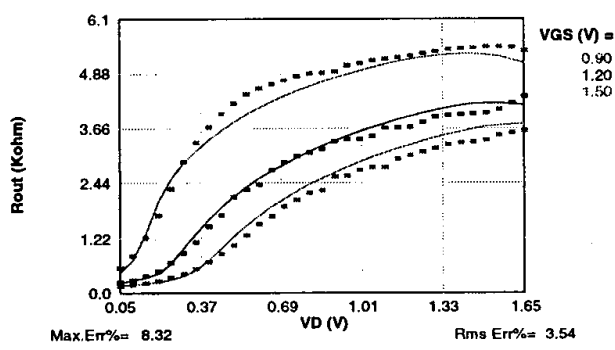

W5L025-1.IV W/ $=5.00 / 0.25 \quad T=27 C$ VB=0.00 V

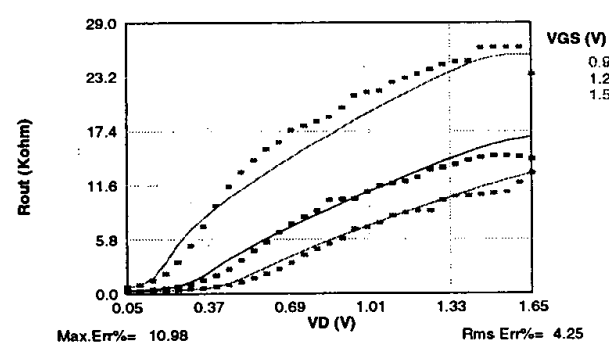

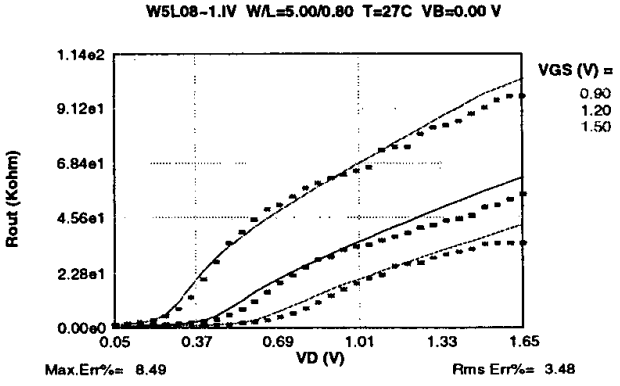

W5L10.IV WR $=5,00 / 40.00$ T=27C VB=0.00 V

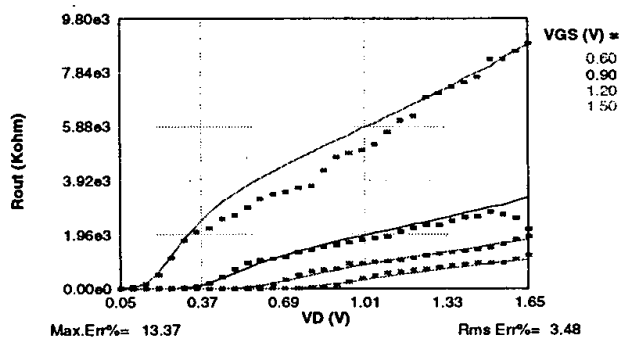

Fig. 13. On the left, the equivalent circuit of the implementation of proposed model. On the right is the fitting of measured $R_{\text {out }}$ of $0.18 \mu \mathrm{m}$ technology using the model implemented. 12 devices with gate lengths from $0.15 \mu \mathrm{m}$ to $10 \mu \mathrm{m}$ are fitted using a single set of parameters without binning. The maximum error is $17.0 \%$ and the RMS error is $4.9 \%$. A new $V_{d s a t}$ model and CLM $R_{o u t}$ model were also implemented for the fitting.

\section{5 .4}

